\title{
Proximity Induced Enhancement of the Curie Temperature in Hybrid Spin Injection Devices
}

\author{
C. Song, M. Sperl, M. Utz, M. Ciorga, G. Woltersdorf, D. Schuh, D. Bougeard, C. H. Back, and D. Weiss* \\ Institut für Experimentelle und Angewandte Physik, Universität Regensburg, 93040 Regensburg, Germany
}

(Received 15 March 2011; published 26 July 2011)

\begin{abstract}
We investigate the increase of the Curie temperature $T_{C}$ in a lateral spin injection geometry where the ferromagnetic (Ga,Mn)As injector and detector contacts are capped by a thin iron film. Because of interlayer coupling between $\mathrm{Fe}$ and $(\mathrm{Ga}, \mathrm{Mn}) \mathrm{As} T_{C}$ gets enhanced by nearly $100 \%$ for the thinnest (Ga,Mn)As films. The use of the proximity effect might pave the way for practical implementation of spintronic devices.
\end{abstract}

DOI: 10.1103/PhysRevLett.107.056601

PACS numbers: 72.25.-b, 75.30.Et, 75.50.Pp, 85.75.-d

The diluted magnetic semiconductor (DMS) (Ga,Mn)As [1] stands out as a seminal spintronic material due to its high spin polarization $[2,3]$ and superior interfacing properties with nonmagnetic semiconductors. This opens a way to semiconducting spintronic devices with functionalities such as nonvolatility and the additional spin degree of freedom [4]. However, the persistently low Curie temperature of DMS has been an obstacle for the integration of DMS into electronic devices. It has been recently shown that the presence of a thin layer of Fe couples the magnetic moments of $\mathrm{Fe}$ and $\mathrm{Mn}$ in $\mathrm{Fe} /(\mathrm{Ga}, \mathrm{Mn})$ As bilayers up to room temperature (RT), thus well exceeding the $T_{C}$ of $(\mathrm{Ga}, \mathrm{Mn}) \mathrm{As}$ [5-7]. This proximity polarization leads to an antiparallel coupling of the $\mathrm{Fe}$ and $\mathrm{Mn}$ moments within an interfacial region of a few nm thickness in the $(\mathrm{Ga}, \mathrm{Mn}) \mathrm{As}$ film. In addition, exchange bias has been observed in the $\mathrm{Fe} /(\mathrm{Ga}, \mathrm{Mn})$ As bilayer system at lower temperatures $T$ [7]. Similar exchange bias has been obtained in MnAs/ (Ga, Mn)As bilayers [8-10]. Differently, NiFe/(Ga, Mn)As bilayers switch their magnetization independently [11] and also $\mathrm{MnTe} /(\mathrm{Ga}, \mathrm{Mn})$ As heterojunctions did not show exchange bias [12]. Here we use the interlayer coupling between $\mathrm{Fe}$ and $(\mathrm{Ga}, \mathrm{Mn}) \mathrm{As}$ to explore its impact on the operation temperature of $(\mathrm{Ga}, \mathrm{Mn}) \mathrm{As}$ based spin injector or detector contacts in a lateral transistorlike geometry. The experiments described below demonstrate enhanced spin injection temperatures measured in an all-electrical fashion.

The heterojunctions were grown by molecular-beamepitaxy (MBE) on (001) GaAs substrates and consist of a $1000 \mathrm{~nm}$ thick $n$-type transport channel, doped with $4 \times 10^{16} \mathrm{~cm}^{-3} \mathrm{Si}$, a $15 \mathrm{~nm}$ thin $n \rightarrow n^{+}$GaAs transition layer $\left(n^{+}=5 \times 10^{18} \mathrm{~cm}^{-3}\right), 8 \mathrm{~nm} n^{+}$-GaAs, and $2.2 \mathrm{~nm}$ low-temperature (LT)-grown $\mathrm{Al}_{0.36} \mathrm{Ga}_{0.64} \mathrm{As}$, serving as a diffusion barrier, followed by LT-grown $\mathrm{Ga}_{0.95} \mathrm{Mn}_{0.05} \mathrm{As}$, the thickness of which was chosen between 5 and $20 \mathrm{~nm}$. The highly doped (Ga, Mn)As/GaAs pn junction forms an Esaki diode $[13,14]$. In a next step the wafers were transferred, without breaking vacuum, into an attached metal-MBE chamber, where $2 \mathrm{~nm}$ of $\mathrm{Fe}$, corresponding to 14 monolayers (MLs), were epitaxially grown at RT, and finally covered by $4 \mathrm{~nm}$ (20 MLs) of Au. The lateral channel of the devices was made by standard lithographic techniques. Electron beam lithography was used to pattern the $\mathrm{Fe} /(\mathrm{Ga}, \mathrm{Mn})$ As injector and detector contacts, oriented along the [110] direction, i.e., the easy axis of Fe. A schematic of the sample layout is shown in Fig. 1. A high resolution transmission electron micrograph of the $\mathrm{Au} / \mathrm{Fe} /(\mathrm{Ga}, \mathrm{Mn}) \mathrm{As}$ stack is included, demonstrating the defect-free epitaxy and sharp interfaces on a monolayer scale. Figure 2(a) presents a scanning electron micrograph of the central region of one of the devices. The contacts have a center-to-center spacing of $6 \mu \mathrm{m}$ and a width of 0.4 or $2 \mu \mathrm{m}$, and the two reference contacts 1 and 6 are separated by $\sim 350 \mu \mathrm{m}$ distance from the central contacts. The transport measurements were carried out between $1.8 \mathrm{~K}$ and $\sim 80 \mathrm{~K}$ in a vector magnet system, allowing us to apply a magnetic field $\mu_{0} H$ up to $1 \mathrm{~T}$ in any direction. A field offset due to the hysteresis of the superconducting coils was subtracted so that $H=0$ coincides with the depolarization peak. Voltages with $H$ applied in-plane were either measured in nonlocal [14-18] or three-terminal

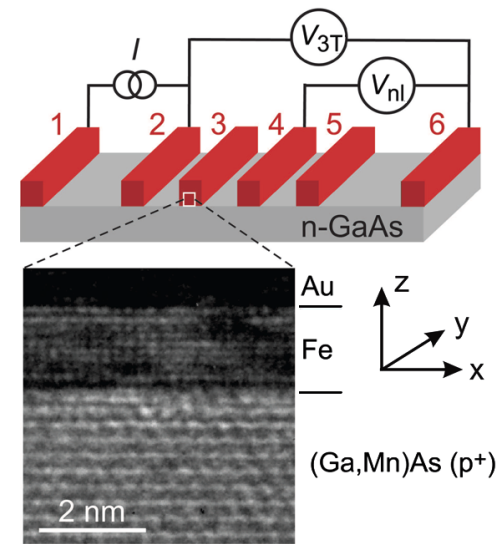

FIG. 1 (color online). Schematic sample design with $\mathrm{Fe} /(\mathrm{Ga}, \mathrm{Mn})$ As contacts shown in red. The voltage is either measured in 3-T or nonlocal configuration. The inset shows a transmission electron micrograph of one of our $\mathrm{Au} / \mathrm{Fe} /(\mathrm{Ga}, \mathrm{Mn}) \mathrm{As}$ interfaces. 
(3-T) configuration [19-21], sketched in Fig. 1. The nonlocal voltage $V_{\mathrm{nl}}$ measured, e.g., between detector $j(j=3$, 4 and 5) and reference contact 6 when a current flows between contacts 1 and $2\left(I_{21}=50 \mu \mathrm{A}\right.$ in our measurements) is a measure of the spin accumulation underneath contact $j$ and given by [16]

$$
V_{\mathrm{nl}}= \pm \frac{1}{2 S} P^{2} I \lambda_{\mathrm{sf}} \rho_{\mathrm{GaAs}} \exp \left(-d / \lambda_{\mathrm{sf}}\right)
$$

with the spin injection (detection) efficiency $P$ of the injector (detector) contact, the current $I$ through the injector, and $\rho_{\mathrm{GaAs}}, \lambda_{\mathrm{sf}}, d$ and $S$ are resistivity, spin diffusion length, injector-detector distance and the cross-section area of the $n$ GaAs channel, respectively. The 3-T voltage contains additional information on the interface resistance and tunnelling anisotropic magnetoresistance $[14,20]$. To unambiguously confirm spin injection we performed 3-T and nonlocal measurements also in a perpendicular $H$ field, i.e., in Hanle geometry [14-18].

We have investigated 3-T, nonlocal and Hanle configuration for Fe-covered $5 \mathrm{~nm}, 10 \mathrm{~nm}, 15 \mathrm{~nm}$, and $20 \mathrm{~nm}$ thick (Ga,Mn)As films at various $T$. In the following, we focus primarily on data obtained from the thickest $(20 \mathrm{~nm})$ and the thinnest Fe-covered $(\mathrm{Ga}, \mathrm{Mn}) \mathrm{As}(5 \mathrm{~nm})$ as these data cover all the central features found in the experiments. To demonstrate the effect of the Fe overlayer on spin injection we first show in Fig. 2(b) the nonlocal spin-valve (SV) signal, using the geometry sketched in Fig. 1, of a $20 \mathrm{~nm}$ (Ga,Mn)As reference sample without Fe cap. This reference sample has been fabricated from the same wafer as the Fe coated $20 \mathrm{~nm}$ thick (Ga,Mn)As by covering one half of the $(\mathrm{Ga}, \mathrm{Mn}) \mathrm{As} / \mathrm{GaAs}$ wafer with a shutter while depositing $\mathrm{Fe}$ and $\mathrm{Au}$ on the other half. The $H$-field was oriented along [100], the easy axis of $(\mathrm{Ga}, \mathrm{Mn}) \mathrm{As}$, and the data were taken at $50 \mathrm{~K}$, shown without subtracting the characteristic offset, often found in nonlocal SV experiments $[14,17]$. The downward spike at zero field is found in all data at lower $T$ and is caused by the polarized nuclei generated by the spin-polarized current across the Esaki diode $[14,22,23]$. This we conclude from the $T$ dependence and from the long time constants, which closely follow those described for spin injection from $\mathrm{Fe}$ into $n$-GaAs $[22,23]$. The existence of this low field structures due to hyperfine interaction complicates the identification of the first switching field close to $H \sim 0$ if, e.g., $H$ is swept from positive to negative values (black trace). After reaching a minimum value in Fig. 2(b) the $(\mathrm{Ga}, \mathrm{Mn}) \mathrm{As}$ switches abruptly at $\mu_{0} H=6.5 \mathrm{mT}$ and the magnetization in the injector and detector contacts is again parallel. This behavior is quite characteristic for spin injection experiments involving (Ga,Mn)As [14].

The presence of a few MLs of epitaxial Fe on top of $20 \mathrm{~nm}(\mathrm{Ga}, \mathrm{Mn})$ As has a profound effect on both 3-T and nonlocal SV measurements, as displayed in Fig. 2(c). In the top row of Fig. 2(c) major hysteresis loops are shown. In addition to the switching at $\sim 6 \mathrm{mT}$ of the $(\mathrm{Ga}, \mathrm{Mn}) \mathrm{As}$, the (a)
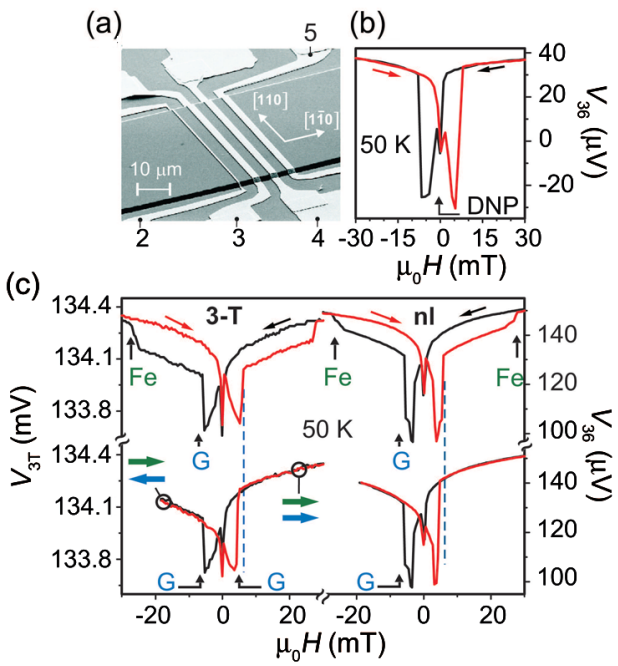

FIG. 2 (color online). (a) Electron micrograph of the central region of the device showing injector and detector stripes across the $50 \mu \mathrm{m}$ wide GaAs channel. (b) Nonlocal voltage $V_{36}$ measured at $d=6 \mu \mathrm{m}$ distance from the injector contact 2 for a reference sample without $\mathrm{Fe}$ on the $20 \mathrm{~nm}(\mathrm{Ga}, \mathrm{Mn}) \mathrm{As}$ film at $50 \mathrm{~K}$. The downward cusp at zero field is due to dynamical nuclear polarization (DNP). The sweep direction is given by arrows. (c) Major loops (top row, $\mu_{0} H$ between $\pm 200 \mathrm{mT}$ ) and minor loops (bottom row, $\mu_{0} H$ between $+200 \mathrm{mT}$ and $-18 \mathrm{mT}$ ) of the $3-\mathrm{T}$ and nonlocal signal for the $\mathrm{Fe} / 20 \mathrm{~nm}$ $(\mathrm{Ga}, \mathrm{Mn})$ As sample at $50 \mathrm{~K}$. Switching fields for Fe and $(\mathrm{Ga}$, $\mathrm{Mn}$ )As (abbreviated by $\mathrm{G}$ ) are marked by arrows. Within a minor loop the magnetization direction of $(\mathrm{Ga}, \mathrm{Mn})$ As switches (thick blue arrow) whereas the direction of the $\mathrm{Fe}$ magnetization remains unchanged (thick green arrow). The shift of the switching field due to exchange bias is indicated by the dashed vertical lines.

switching of the Fe layer at $\sim 26 \mathrm{mT}$ is clearly recognizable in the major loop of both, 3-T and nonlocal measurements [24]. The minor loops plotted in the bottom row of Fig. 2(c) were obtained by the following procedure: first the magnetization of $\mathrm{Fe}$ and $(\mathrm{Ga}, \mathrm{Mn}) \mathrm{As}$ was saturated at $+200 \mathrm{mT}$, then $\mu_{0} \mathrm{H}$ was swept down and stopped at $-18 \mathrm{mT}$, just before reaching the switching field of $\mathrm{Fe}$ at $-26 \mathrm{mT}$. Finally, the field was ramped up again (red trace) back to $+200 \mathrm{mT}$. The most eminent feature observed here is the field shift of the switching in the minor loops as marked by the dashed vertical lines in Fig. 2(c). This shift of the minor loop to the left, opposite to the magnetization of the $\mathrm{Fe}$ overlayer, indicates a negative exchange bias [8-10]. This negative exchange bias is seen in the minor loops of both, 3-T and nonlocal measurement (lower panel), contrasting the symmetric switching of the major loop (upper panel). The bias field of $\sim 0.8 \mathrm{mT}$ extracted from spin injection measurements is also observed in the minor loops of corresponding unpatterned films using superconducting quantum interference device (SQUID) magnetometry. The separate switching fields for $(\mathrm{Ga}, \mathrm{Mn})$ As and $\mathrm{Fe}$ together with the observed exchange bias 
suggest that in the immediate vicinity of the $\mathrm{Fe} /(\mathrm{Ga}, \mathrm{Mn}) \mathrm{As}$ interface a thin layer of $(\mathrm{Ga}, \mathrm{Mn}) \mathrm{As}$ is antiferromagnetically pinned to the magnetically hard $\mathrm{Fe}$ layer due to the proximity effect [5-7], but the biased bulk of the $(\mathrm{Ga}, \mathrm{Mn})$ As layer is free to rotate in a reversed $H$ field. A similar scenario was found in $\mathrm{MnAs} /(\mathrm{Ga}, \mathrm{Mn}) \mathrm{As}$ bilayers $[9,10]$. The maximum temperature at which we observe spin injection in Hanle and nonlocal measurements for the $20 \mathrm{~nm}$ device is $\sim 75 \mathrm{~K}$, close to the $T_{C}$ of $82 \mathrm{~K}$ measured by SQUID magnetometry. No enhancement of $T_{C}$ due to the presence of $\mathrm{Fe}$ is found.

The situation changes dramatically when the thickness of the $(\mathrm{Ga}, \mathrm{Mn})$ As is reduced to $5 \mathrm{~nm}$. Corresponding data for $H$ aligned along [110] are presented in Fig. 3. In the top panels of Fig. 3 we compare the nonlocal SV signals $V_{36}$ of a Fe-covered sample in Figs. 3(a)-3(d) with the ones of a reference sample without Fe [Figs. 3(e)-3(g)] between 1.8 and $70 \mathrm{~K}$. Remarkably, no separate switching field for $\mathrm{Fe}$ is observed any more, i.e., $\mathrm{Fe}$ and $(\mathrm{Ga}, \mathrm{Mn}) \mathrm{As}$ switch together. This joint switching is also seen in SQUID measurements as will be discussed below. For the signal obtained at $1.8 \mathrm{~K}$ the (upper) switching field, defined by the maximum slope (peak of derivative) of $V_{36}$, is $\sim 37 \mathrm{mT}$, which is larger than the switching field of the $(\mathrm{Ga}, \mathrm{Mn}) \mathrm{As}$ reference and close to the one of $\mathrm{Fe}$. The nonlocal signal
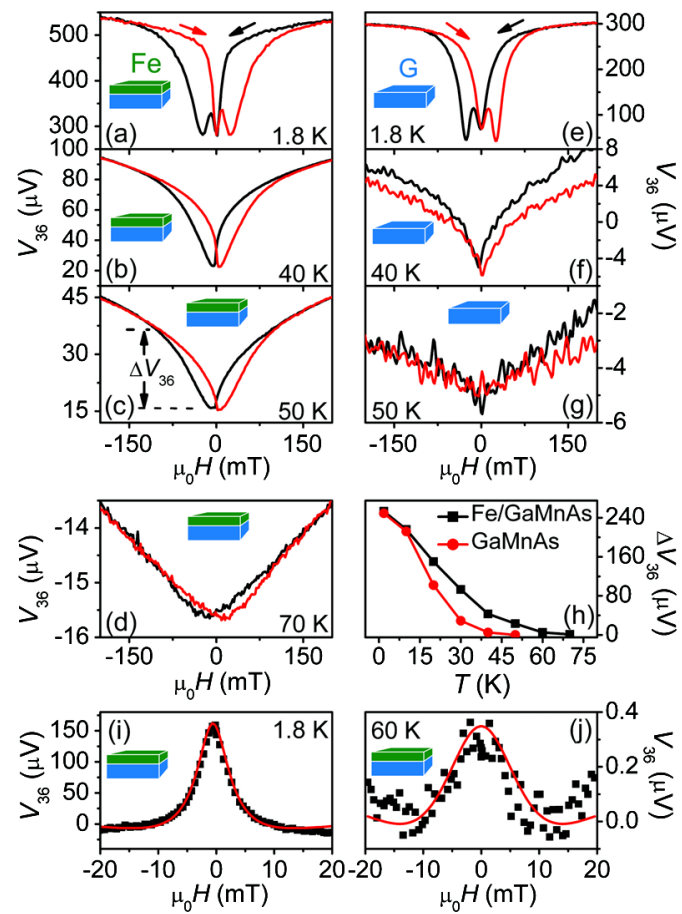

FIG. 3 (color online). Nonlocal signal $V_{36}$ for contacts with (a)-(d) and without (e)-(g) $2 \mathrm{~nm}$ Fe overlayer on $5 \mathrm{~nm}$ (Ga,Mn) As at different temperatures. (h) Signal height $\Delta V_{36}$ vs temperature for $\mathrm{Fe} / 5 \mathrm{~nm}(\mathrm{Ga}, \mathrm{Mn}) \mathrm{As}$ and its reference samples. Nonlocal Hanle signal (black squares) and corresponding fits (red line) after offset subtraction at 1.8 and $60 \mathrm{~K}$ for the $\mathrm{Fe} / 5 \mathrm{~nm}$ $(\mathrm{Ga}, \mathrm{Mn})$ As device are presented in (i) and (j), respectively. measured at larger distances from the injector, $V_{46}$ and $V_{56}$ show the same qualitative behavior, but with a reduced overall magnitude. The amplitude of $V_{\mathrm{nl}}$ decays exponentially with the injector-detector distance $d$ in good agreement with Eq. (1), yielding $\lambda_{\text {sf }} \sim 5.2 \mu \mathrm{m}$. Here, $\lambda_{\text {sf }}$ is larger than the value reported in Ref. [14], which can be ascribed to the lower doping of the present $n$-GaAs channel.

We now turn towards the $T$ dependence of the nonlocal signals. At $50 \mathrm{~K}$ the sample with the Fe layer on top still shows a pronounced nonlocal signal [Fig. 3(c)] whereas the corresponding reference sample in Fig. 3(g) shows no hysteretic signal any more. A direct comparison of the amplitude of the nonlocal signal $\Delta V_{36}$, as defined in Fig. 3(c), is shown in Fig. 3(h). At $1.8 \mathrm{~K}, \Delta V_{36}$ of both types of devices is practically the same, but $\Delta V_{36}$ of the reference device decays much faster with increasing $T$. Measurements on the Fecovered sample exhibit a resolvable nonlocal hysteretic SV signal up to $T \sim 70 \mathrm{~K}$, shown in Fig. 3(d), while the nonlocal signal of the reference vanishes just above $40 \mathrm{~K}$. We note that the enhanced $T_{C}$ observed in our spin injection experiments cannot be explained by unintended annealing during sample preparation [10]: (i) the Fe layer and Au cap are grown at RT thus preventing annealing of the (Ga,Mn)As; (ii) both, $\mathrm{Fe} /(\mathrm{Ga}, \mathrm{Mn}) \mathrm{As}$ and $(\mathrm{Ga}, \mathrm{Mn})$ As reference devices are fabricated simultaneously in the same chamber from the same wafer; (iii) the absence of any $T_{C}$ enhancement in the $\mathrm{Fe} / 20 \mathrm{~nm}(\mathrm{Ga}, \mathrm{Mn})$ As device confirms the absence of annealing effects in our devices.

In addition to the nonlocal SV measurements we have explored spin precession and dephasing by employing the Hanle effect [14-18]. Typical Hanle signals from the Fecovered samples measured at 1.8 and $60 \mathrm{~K}$ are shown in Figs. 3(i) and 3(j), respectively. Analyzing the data within a drift-diffusion model [14-18], we obtain $P=0.38, \tau_{s}=$ $8.7 \mathrm{~ns}$ and $\lambda_{\text {sf }}=5.3 \mu \mathrm{m}$ at $1.8 \mathrm{~K}$. Note that the latter value is consistent with the one extracted from the exponential decay of the nonlocal SV signal. With increasing $T$, the decreasing amplitude and increasing width of the Hanle curve reflects decreasing values of $P, \tau_{s}$, and $\lambda_{\text {sf }}$. These quantities assume values of $0.12,2.2 \mathrm{~ns}$, and $1.5 \mu \mathrm{m}$ at $60 \mathrm{~K}$.

The spin injection measurements have revealed a significant increase of $T_{C}$ from $\sim 40 \mathrm{~K}$ to $\sim 70 \mathrm{~K}$ due to the presence of a few MLs of Fe on top of $5 \mathrm{~nm}(\mathrm{Ga}, \mathrm{Mn})$ As. We now address the question whether an analogous increase can also be observed by SQUID magnetometry. For these experiments large areas of $4 \mathrm{~mm} \times 4 \mathrm{~mm}$ $\mathrm{Fe} /(\mathrm{Ga}, \mathrm{Mn})$ As samples are needed. In Fig. 4(a) the field dependence of the magnetic moment $m(H)$ for $\mathrm{Fe} / 5 \mathrm{~nm}$ (Ga,Mn)As is compared to that of the corresponding reference sample for $H$ aligned along [110]. For that the $T$-independent diamagnetic contribution, obtained at high fields, was subtracted from the hysteresis. A single switching event at $\pm 33 \mathrm{mT}$ confirms the joint switching of Fe and $(\mathrm{Ga}, \mathrm{Mn}) \mathrm{As}$, already seen in the spin injection experiments 

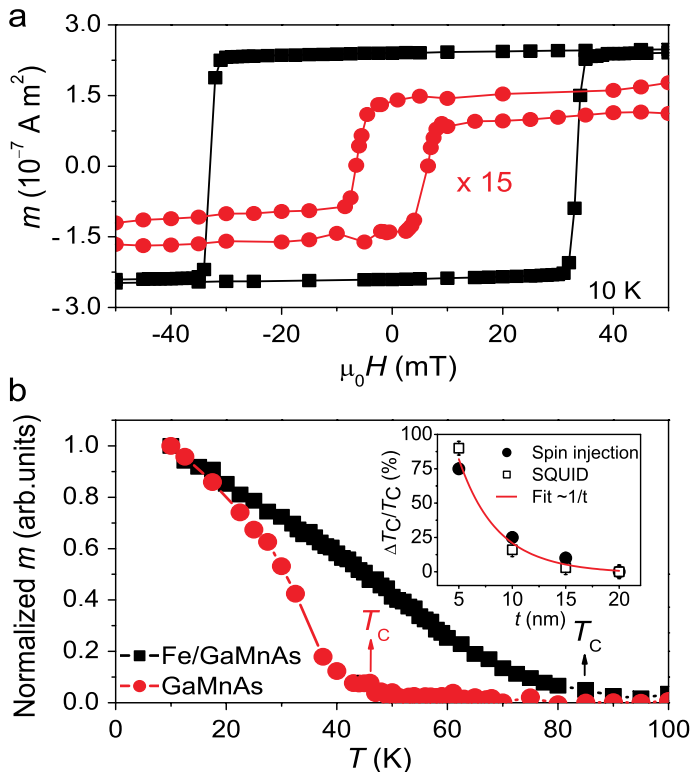

FIG. 4 (color online). (a) SQUID measurement of $m(H)$ for $\mathrm{Fe} / 5 \mathrm{~nm}(\mathrm{Ga}, \mathrm{Mn}) \mathrm{As}$ and the corresponding (Ga,Mn)As reference along [110] at $10 \mathrm{~K}$. (b) $m(T)$ at a constant field of $\mu_{0} H=$ $10 \mathrm{mT}$ along [110]. $T_{C}$ of $(\mathrm{Ga}, \mathrm{Mn})$ As is taken at the inflection point of the measured $m(T)$ curves. The inset displays $\Delta T_{C} / T_{C}$ obtained from spin injection and SQUID data for different (Ga, Mn)As thickness $t$.

displayed in Fig. 3. Note that the associated switching fields cannot directly be compared as coercive fields of extended films (for SQUID) and of micropatterned contact strips (for spin injection) are different $[25,26]$. To determine $T_{C}$ with SQUID magnetometry, we measured the $T$ dependence of the magnetic moment $m(T)$ at $10 \mathrm{mT}$, shown in Fig. 4(b) [27]. $m(T)$ curves taken with SQUID show an increase of $T_{C}$ from $(45 \pm 3) \mathrm{K}$ for the reference sample to $(85 \pm 4) \mathrm{K}$ for $\mathrm{Fe} / 5 \mathrm{~nm}(\mathrm{Ga}, \mathrm{Mn})$ As. The increased $T_{C}$ measured by spin injection, shown in Fig. 3(h), and SQUID, displayed in Fig. 4, is qualitatively and quantitatively very similar and constitutes the main finding of our work. An enhanced $T_{C}$ is also found for $(\mathrm{Ga}, \mathrm{Mn})$ As film thicknesses $t$ up to $15 \mathrm{~nm}$. The resulting normalized $T_{C}$ enhancement, $\Delta T_{C} / T_{C}$ with $\Delta T_{C}=$ $T_{C}$ ( with $\left.\mathrm{Fe}\right)-T_{C}$ (reference) for different $t$ is summarized in the inset of Fig. 4(b). $\Delta T_{C} / T_{C}$ drops rapidly with $\Delta T_{C} / T_{C} \propto 1 / t$ reflecting the reduced exchange coupling with increasing distance $t$ from the $\mathrm{Fe} /(\mathrm{Ga}, \mathrm{Mn}) \mathrm{As}$ interface [28]. We conclude by noting that the observed maximum increase on the order of $\sim 100 \%$ demonstrates that the proximity effect [5-7] effectively boosts the operation temperature of $(\mathrm{Ga}, \mathrm{Mn}) \mathrm{As}$ injector and detector contacts thus promising RT operation if $T_{C}$ of very thin $(\mathrm{Ga}, \mathrm{Mn}) \mathrm{As}$ layers can reach values of around $190 \mathrm{~K}$, already achieved in thicker films [29].

This work has been supported by the Deutsche Forschungsgemeinschaft (DFG) via SFB689. C.S. is grateful for the support of Alexander von Humboldt
Foundation. We thank M. Soda and J. Zweck for providing the TEM image in Fig. 1 and A. Einwanger for technical support.

*dieter.weiss@physik.uni-regensburg.de

[1] T. Dietl, Nature Mater. 9, 965 (2010).

[2] J. G. Braden, J. S. Parker, P. Xiong, S. H. Chun, and N. Samarth, Phys. Rev. Lett. 91, 056602 (2003).

[3] Van Dorpe et al., Appl. Phys. Lett. 84, 3495 (2004).

[4] D. D. Awschalom and M. E. Flatté, Nature Phys. 3, 153 (2007).

[5] F. Maccherozzi et al., Phys. Rev. Lett. 101, 267201 (2008).

[6] M. Sperl et al., Phys. Rev. B 81, 035211 (2010).

[7] K. Olejnik et al., Phys. Rev. B 81, 104402 (2010).

[8] M. Zhu et al., Appl. Phys. Lett. 91, 192503 (2007).

[9] M. Zhu, M. J. Wilson, P. Mitra, P. Schiffer, and N. Samarth, Phys. Rev. B 78, 195307 (2008).

[10] M. J. Wilson et al., Phys. Rev. B 81, 045319 (2010).

[11] S. Mark et al., Phys. Rev. Lett. 103, 017204 (2009).

[12] J. K. Furdyna, X. Liu, Y. Sasaki, S. J. Potashnik, and P. Schiffer, J. Appl. Phys. 91, 7490 (2002).

[13] M. Kohda, Y. Ohno, K. Takamura, F. Matsukura, and H. Ohno, Jpn. J. Appl. Phys. 40, L1274 (2001).

[14] M. Ciorga et al., Phys. Rev. B 79, 165321 (2009).

[15] M. Johnson and R. H. Silsbee, Phys. Rev. Lett. 55, 1790 (1985).

[16] F. J. Jedema, H. B. Heersche, A. T. Filip, J. J. A. Baselmans, and B.J. van Wees, Nature (London) 416, 713 (2002).

[17] X. Lou et al., Nature Phys. 3, 197 (2007).

[18] O. M. J. van't Erve et al., Appl. Phys. Lett. 91, 212109 (2007).

[19] X. Lou et al., Phys. Rev. Lett. 96, 176603 (2006).

[20] M. Tran et al., Phys. Rev. Lett. 102, 036601 (2009).

[21] S. P. Dash, S. Sharma, R. S. Patel, M. P. de Jong, and R. Jansen, Nature (London) 462, 491 (2009).

[22] G. Salis, A. Fuhrer, and S. F. Alvarado, Phys. Rev. B 80, 115332 (2009).

[23] M. K. Chan et al., Phys. Rev. B 80, 161206(R) (2009).

[24] The emergence of a kink in $V_{3 T}$ and $V_{36}$ when the Fe layer reverses magnetization is likely due to twisting of the exchange spring which forms at the $\mathrm{Fe} /(\mathrm{Ga}, \mathrm{Mn}) \mathrm{As}$ interface (see Refs. $[9,10]$ ) which can affect the magnetization orientation at the (Ga, Mn)As/GaAs interface and thus the detected spin polarization $P$.

[25] W. Kipferl, M. Dumm, M. Rahm, and G. Bayreuther, J. Appl. Phys. 93, 7601 (2003).

[26] F. Hoffmann et al., Phys. Rev. B 80, 054417 (2009).

[27] To separate the $T$ dependence of Fe and (Ga,Mn)As, $m(T)$ of Fe was fitted well above $T_{C}$ of (Ga,Mn)As with Bloch's $T^{3 / 2}$ law. Subsequently, the corresponding $T$ dependence was subtracted from the raw data. For the $(\mathrm{Ga}, \mathrm{Mn}) \mathrm{As}$ reference sample the $T$ independent diamagnetic background above $T_{C}$ is subtracted from the raw data.

[28] E. Z. Meilikhov and R. M. Farzetdinova, J. Phys. Conf. Ser. 200, 032045 (2010).

[29] L. Chen et al., Appl. Phys. Lett. 95, 182505 (2009). 